\title{
Linear-type Orbital Floor Fracture with or without Muscle Involvement
}

Hiroki Yano, M.D., Yuichi Suzuki, M.D., Hiroshi Yoshimoto, M.D., Ritsuko Mimasu, M.D., and Akiyoshi Hirano, M.D.

Department of Plastic and Reconstructive Surgery, Nagasaki University School of Medicine.

\section{Address correspondence to:}

Hiroki Yano, M.D.

Department of Plastic \& Reconstructive Surgery, Nagasaki University School of Medicine, 1-7-1 Sakamoto, Nagasaki 852-8102, Japan

Fax: +81-95-819-7330 Tel: +81-95-819-7327

e-mail: h-yano@net.nagasaki-u.ac.jp

Acknowledgment: This work was supported in part by a Grant-in-Aid for Scientific Research from Nagasaki University, Japan. 


\section{Abstract}

The indications for surgical repair of the orbital blowout fracture are controversial. One reason may be case variation among fracture types. We therefore focused on linear-type blowout fractures in this study. The study included 22 consecutive cases of linear-type floor fractures. Demographics, clinical and CT findings, surgical timing, postoperative course, and outcome were evaluated. Surgery was performed in 14 cases for diplopia but not enophthalmos. Five cases with severe vertical diplopia were defined as "missing rectus" by CT findings. Residual diplopia remained in two cases with "missing rectus", while the other 20 cases completely recovered eye motility. In the 9 cases without muscle entrapment, diplopia disappeared within four weeks after operation. However recovery in cases with "missing rectus" took more than one month. Thus the CT finding with or without muscle involvement was crucial for linear-type blowout fractures. A comprehensive and timely decision based on clinical and radiological findings is indispensable for satisfactory management as well 
as postoperative rehabilitation.

Key words; blowout fracture; linear fracture; missing rectus; postoperative rehabilitation; Bell's phenomenon. 
Management of orbital blowout fracture was relatively conservative, ${ }^{1,2}$ but has recently shifted to favor early surgical repair. ${ }^{3-5}$ In this decade, it has been widely accepted that surgical intervention should be performed early in cases with "white-eyed" blowout fractures, severe oculocardiac reflex, and entrapped orbital contents. ${ }^{6}$ Surgical procedures at a later stage cannot prevent ischemic damage and the formation of excess scar tissue causes postoperative complication. ${ }^{3}$ There has more recently been a return to the conservative view of surgery, ${ }^{7,8}$ rendering its indication and timing unclear. These fluctuations in management may result from the pathological complexity of the blowout fracture. Surgical repair for this injury aims to reinstate the orbit with enophthalmos and to restore diplopia. Enophthalmos may result from expansion of the orbit and/or atrophy of the orbital content. ${ }^{9}$ Diplopia may result from deformity of the orbit (causing positional changes of the muscles), ${ }^{10,11}$ adhesive impingement of the content (disturbing ocular excursion), ${ }^{2,11}$ and impairment of the contracting muscle (resulting from neurogenic or myogenic palsy). ${ }^{11}$ Since living 
organisms have the adaptability to compensate for functional defects, the problems noted above, especially diplopia, may be ameliorated with time.

Because the pathology of blowout fractures varies among cases, management cannot be determined solely by the timing of surgery. The indication for surgery must also be defined according to clinical findings and fracture types. ${ }^{12}$ Therefore, the aim of this study was to bring the management concept into sharper focus for linear-type blowout fractures, especially with regard to postoperative course. We attempt to elucidate pathologic natures of this type of fracture and propose a postoperative remedy.

\section{Patients and Methods}

The medical records of 103 consecutive cases of pure-type blowout fractures presented in our department from 2002 to 2007 were examined. Twenty-two cases were classified as linear-type fractures of the orbital floor by CT findings. Demographics, clinical and CT findings, 
surgical timing, purpose and procedure, postoperative course, and outcome were explored. In this study, "missing rectus" denoted cases for which coronal CT findings revealed minimal or no inferior rectus muscle density that could be confirmed above the floor. ${ }^{13,14}$ For linear fractures, "missing rectus" indicates that the muscle is firmly and tightly entrapped in the fracture site and herniated to the sinus.

Surgical procedure: Surgery was electively applied depending on the clinical and CT findings ${ }^{12}$. The periosteum was sharply incised and retracted from the rim through an extended subciliary incision. After a linear fracture with entrapment of orbital contents was identified, the herniated tissue was carefully retrieved by cutting out the floor with a fine osteotome. So as not to further damage the tissue, we did not withdraw it from the slit but rather scooped the herniated tissue out from the hole. The orbit was then reconstructed by bone graft. Before skin closure, the release of impingement was confirmed by comparing pre- and postoperative forced duction tests, in order to avoid iatrogenic gaze restriction. Finally, the 
incised periosteum was sutured, the skin was closed, and a drain was set in place.

Postoperative rehabilitation: Two or three days after the operation, as swelling and pain gradually disappeared, patients could usually obtain a focused field in binocular vision, although this field might be narrow in the beginning. Aftercare was then initiated to expand the focused field; for example, chasing a slowly moving finger to the periphery of the field and gazing at a distant point while bending and stretching the neck. However, in severe or juvenile patients, it was sufficiently difficult to exercise binocular vision at the start, so a monoculus was applied to the unaffected eye. This method allowed patients to elicit motion of the affected eye in the early stages, while we paid close attention to the possible cause of amblyopia.

Data analysis: Ocular motility was graded on five scales. ${ }^{15}$ The correlation coefficient for upgaze and downgaze preoperative motility was obtained using simple regression analysis. Differences in preoperative motility, with 
or without missing rectus in CT findings, were analyzed in each upgaze and downgaze with the use of the Mann-Whitney $U$ test. Simple comparisons of time to surgery, with or without missing rectus, were performed using Student's $t$-test. Statistical analysis was applied using Microsoft Excel 2003 (http://office.microsoft.com), with Statcel2 add-in software (http://www.oms-publ.co.jp/ex101/howto01.html).

\section{Results}

For the 22 linear-type blowout fractures, the average age of patients was 14.3 years, ranging from 6-45 years. Eighteen patients were male and four were female. Fractures were due to sports injuries in nine patients, followed by falling in six, assault in four, and traffic accidents in three. Average time from injury to presentation was 4.05 days, ranging from 0-20 days. Four cases presented numbness of the infraorbital nerve area. In four cases, except for CT findings, any clinical findings for further treatment were not observed at our presentation. The other four showed 
mild upgaze restriction that spontaneously disappeared over the next few days, while patients were under observation. No enophthalmos was observed in the linear fractures.

Fourteen cases underwent surgical intervention to treat diplopia (Table 1). The extent of preoperative vertical restriction varied among cases (Fig. 1). In contrast to textbooks, upgaze restriction was not clearly dominant as compared with downgaze among our patients $(\mathrm{Y}=1.01 \mathrm{X}+0.30)$. Ocular motility was seriously affected in five cases with "missing rectus" as revealed by $\mathrm{CT}$ findings. Upgaze and downgaze restrictions were more severe in patients with missing rectus, as compared to those without. The average time from injury to surgery was 9.7 days, ranging 0-24 days. There was no difference between patients with or without missing rectus with regard to time to surgery (Table 2). However, in this series, because some cases were referred to us several days after the injury, the timing of surgery varied. No patient worsened after surgery. Residual diplopia remained in only two cases with "missing rectus". Theses cases underwent surgery at 8 
and 24 days. Each of the 20 other cases exhibited completely improved eye movement. The time from surgery to recovery is summarized in Fig.2. Ten out of fourteen surgical cases fully recovered within one month. Cases without missing rectus also completely recuperated within four weeks. Conversely, in the cases with missing rectus, recovery took four or more weeks; gaze restriction remained in two cases. One with residual diplopia is described in the next paragraph. Another showed degeneration in and around the inferior rectus muscle on MRI images six months after surgery (Fig. 3). Perimuscular tissue dissection was performed, but the results did not satisfy the patient.

Surgery was performed in three cases out of four that presented with numbness of the infraorbital nerve. One patient suffered numbness after surgery; the numbness was temporal and disappeared without further intervention.

Case 10, with missing rectus and sequelae (Fig. 4): A six-year-old girl suffered a bruise on her right eye, and was referred to a previous surgeon 
for diplopia 20 days after injury. A surgical correction and bone grafting were performed 24 days after injury, but the vertical restriction of eye movement persisted. The surgeon then referred her to us for further treatment of diplopia three months after surgery. We performed surgery to release the adhesion around the inferior rectus muscle, reconstructed again the floor with a large bone graft, and applied traction to the muscle for a week. Ductions improved, but diplopia continued; however, diplopia was not serious enough to warrant strabismus surgery. The CT images just after injury were checked carefully at a later date, and showed missing rectus with a minimally dislocated linear floor fracture.

Case 14, with missing rectus, without sequelae (Fig. 5): A nine-year-old boy was injured in the right eye after falling from a tree. The patient visited a local ophthalmologic clinic because of severe diplopia without nausea or vomiting. The ophthalmologist referred him to us with suspicion of a blowout fracture. The referral was not urgent, and the patients visited us as a typical outpatient two days after the injury. The vertical restriction of the 
right eye movement was severe and $\mathrm{CT}$ revealed a muscle incarceration within a linear fracture. Emergency surgery was performed to rescue the missing rectus on the same day. Although the forced duction test after bone grafting revealed good excursion, motility of the right eye was initially discouraging. We advised the patient to wear a monoculus on the left eye for one hour, three times a day. Motility was slowly but definitely recovered; recovery was completed at 12 weeks after surgery.

\section{Discussion}

Blowout fractures lead to two disorders; enophthalmos, which impacts esthetics, and diplopia, which impedes function. In general, the former progressively worsens and the later gradually improves over time. The pathology of these two disorders is complicated, and living organisms have the adaptability to compensate for functional defects. We therefore focused on the linear-type blowout fracture.

In this study, surgeries were applied in 14 out of 18 cases with 
diplopia at presentation and almost all were performed for remaining diplopia within two weeks. Surgeries were performed within two weeks because with this type of fracture, diplopia improved little through several days of observation. As many publications about trap door fractures have mentioned, ${ }^{3,16,17}$ the lack of a spontaneous cure can attribute to mechanical interferences, such as entrapment. Entrapment in linear-type blowout fractures may result in changes that are more focally vulnerable than in other types of fractures. Taken together, these results indicate that the primary pathology of linear-type blowout fracture is dyskinesia resulting from adhesive impingement of the content or impairment of the contracting muscle. $^{11}$

Among the cases included in this study, those with 8 or 24 days between the injury and surgery displayed residual diplopia. Other cases with 0,2 , and 5 days between injury and surgery displayed full recovery. Although the critical threshold appears to fall between 5 and 8 days, the degree of injury, as well as the time to surgery, is an important factor that 
determines the severity of residual diplopia. CT images of case 12 did not reveal a fracture but did indicate a missing rectus, ${ }^{18}$ thus muscle damage was estimated to be greater than in case 14 . Interestingly, although diplopia in case 12 disappeared through six-week rehabilitation, diplopia in case 14 did not disappear for twelve weeks. However even in case 12, diplopia took more than four weeks to heal. These results imply that the damage already presents at injury ${ }^{11}$ and early surgery prevents spreading it (ie, scar adhesion) around the fracture. As in myocardial infarction, if damage is minimal and appropriate management is undertaken in a timely fashion, the remaining healthy muscle compensates for damage-related defects. In blowout fractures, fortunately, muscle entrapment in the linear fracture is rare. ${ }^{12,19}$ If muscle entrapment is observed, it involves only limited portions of the muscle, leaving more than half of the muscle belly still intact. ${ }^{18}$ Some advocate early surgery to prevent ischemic alterations of an impinged muscle. $^{13,16}$ However, because muscle ischemia becomes irreversible within hours, it may be nearly impossible to perform the operation after 
definitive diagnosis. ${ }^{3,19}$ We believe that blood flow may not be completely absent in muscles entrapped in blowout fractures. Regions of ischemic necrosis and unstable regions such as the zone of stasis in thermal trauma ${ }^{20}$ may coexist around the fracture site. Therefore, because missing rectus recovery is time-consuming, early surgery and early rehabilitation are indispensable for such fractures. However persistent gaze restriction might be unavoidable for cases with severe muscle damages beyond a critical point even with an urgent surgery. Although surgery for missing rectus is time-intensive, the procedure for linear fractures must be simpler than in other types because dissection around the infraorbital nerve is rarely required and the orbital buttress remains intact in most linear-type fractures. $^{12}$

During the postoperative course of some blowout fractures, a paradoxical eye movement was observed (Fig. 6). This odd phenomenon was observed during the early postoperative phase. After dissolving the impingement in the fracture by surgical intervention, it may result from 
compensation or disturbance of the afferent nervous signaling by stretch receptors $^{21,22}$ in the orbit. In cases with missing rectus, early effective rehabilitation is important for fully recovered ductions as infraduction restriction initially remains serious despite the removal of mechanical interference. When binocular vision exercises are difficult for severely injured or juvenile patients, a monoculus on the unaffected eye during the early phase of postoperative recovery may help to rehabilitate the affected eye motion, while avoiding amblyopia in children.

In any type of blowout fracture including medial wall fractures, vertical gaze, especially upgaze, impairments are more common than lateral gaze restriction. Linear-type or closed trap-door fractures may be frequent in the floor because of structural differences in the paranasal sinuses (ethomoid cells vs. maxillary sinus). This specificity may also be related to Bell's phenomenon at injury (Fig. 7). ${ }^{12}$ Almost all patients with blowout fractures have suffered blunt injuries and are therefore forced to close their eyes firmly just prior to injury. Closing the eyes results in 
supraduction (= Bell's phenomenon), which lengthens the inferior rectus and brings the muscle in front of the eye's equatorial plane. If fracture occurs under the muscle leading to herniation through the fracture site, more damage may occur upon closing the "trap door" when the eye returns to the primary position. The returning force may assist in closing the door which may become lodged and damage itself at the fracture edge. In addition, Iliff et al. reported that the inferior rectus muscle near the apex was delicate in comparison to the denser connective tissue around the muscle at its global insertion. ${ }^{11}$ Some reports have noted muscle swelling on the floor without the identification of missing rectus in CT findings. ${ }^{11,23}$, ${ }^{24}$ The rectus may be damaged upon return to the primary position, similar to degloving injury. This can occur in any fractures with sharp edges. However, because punched out or burst-type fractures may result in orbital decompression in a sense, early reconstruction of the floor in such cases may worsen the swollen muscles by a surgical invasion in addition to post-traumatic stress. ${ }^{12}$ 
In conclusion, in cases without "missing rectus", surgery for diplopia in linear-type blowout fractures can be postponed for several days until swelling has decreased. Because orbital surgeries are technically easier after the swelling has subsided and planned surgeries are preferable with regard to economics considerations. ${ }^{12}$ In cases with "missing rectus", surgery should be performed immediately in order to save unstable regions of the orbital muscle because recovery time and sequelae were correlated to both degree of the damage and time to surgery. A comprehensive and timely decision based on clinical and radiological findings is required for satisfactory management. If surgeons waver in terms of their decision regarding borderline findings, such as in cases with severe vertical restriction but unclear missing rectus on CT images, currently, surgery should be performed immediately for linear-type fractures. Compared with other types of fractures, the linear-type blowout fracture less frequently exhibits spontaneous healing. Surgery will be needed, whether or not the patients are observed for several days, in this type of fractures. 


\section{References}

1) Putterman AM, Stevens T, and Urist MJ. Nonsurgical management of blow-out fractures of the orbital floor. Am J Ophthalmol 1974; 77: 232-239.

2) Koornneef L. Current concepts on the management of orbital blow-out fractures. Ann Plast Surg 1982; 9: 185-200.

3) Grant JH 3rd, Patrinely JR, Weiss AH, et al. Trapdoor fracture of the orbit in a pediatric population. Plast Reconstr Surg 2002; 109: 482-489,.

4) Jordan DR, Allen LH, White J, et al. Intervention within days for some orbital floor fractures: the white-eyed blowout. Ophthal Plast Reconstr Surg 1998; 14: 379-390.

5) Brady SM, McMann MA, Mazzoli RA, et al. The diagnosis and management of orbital blowout fractures: update 2001. Am J Emerg Med 2001; 19:147-154.

6) Burnstine MA. Clinical recommendations for repair of isolated orbital floor fractures: an evidence-based analysis. Ophthalmology 2002; 109:1207-1210.

7) Simon GJ, Syed HM, McCann JD, et al. Early versus late repair of orbital blowout fractures. Ophthalmic Surg Lasers Imaging 2009; 40: 141-148. 
8) Dal Canto AJ and Linberg JV. Comparison of orbital fracture repair performed within 14 days versus 15 to 29 days after trauma. Ophthal Plast Reconstr Surg 2008; 24: 437-443.

9) Whitehouse RW, Batterbury M, Jackson A, et al. Prediction of enophthalmos by computed tomography after 'blow out' orbital fracture. $\mathrm{Br} J$ Ophthalmol 1994; 78: 618-620.

10) Converse JM. Two plastic operations for repair of the orbit following severe trauma and extensive comminuted fracture. Arch Ophthalmol 1944; 31: 323-326.

11) Iliff N, Manson PN, Katz J, et al. Mechanisms of extraocular muscle injury in orbital fractures. Plast Reconstr Surg 1999; 103: 787-799.

12) Yano H, Nakano M, Anraku K, et al. A consecutive case review of orbital blowout fractures and recommendations for comprehensive management. Plast Reconstr Surg 2009; 124: 602-611.

13) Wachler BS, and Holds JB. The missing muscle syndrome in blowout fractures: an indication for urgent surgery. Ophthal Plast Reconstr Surg 1998; 14: 17-18. 
14) Anda S, Elsås T, and Harstad HK. The missing rectus: a CT observation from blow-out fracture of the orbital floor. J Comput Assist Tomogr 1987; 11: 895-897.

15) Criden MR, and Ellis FJ. Linear nondisplaced orbital fractures with muscle entrapment. J AAPOS 2007; 11: 142-147.

16) Okinaka Y, Hara J, and Takahashi M. Orbital blowout fracture with persistent mobility deficit due to fibrosis of the inferior rectus muscle and perimuscular tissue. Ann Otol Rhinol Laryngol 1999; 108:1174-1176.

17) Koornneef L. Orbital septa: anatomy and function. Ophthalmology 1979; 86: 876-880.

18) Yano H, Minagawa T, Masuda K, et al. Urgent rescue of 'missing rectus' in blowout fracture. J Plast Reconstr Aesthet Surg 2009; 62: e301-304.

19) Manson PN, Iliff N and Robertson B. Discussion; Trapdoor fracture of the orbit in a pediatric population. Plast Reconstr Surg 2002; 109: 490-495.

20) Jackson DM. The diagnosis of the depth of burning. Br J Surg 1953; 40: 588-596. 
21) Dancause N, Taylor MD, Plautz EJ, et al. A stretch reflex in extraocular muscles of species purportedly lacking muscle spindles. Exp Brain Res 2007; $180 ; 15-21$.

22) Demer JL. Current concepts of mechanical and neural factors in ocular motility. Curr Opin Neurol 2006; 19; 4-13.

23) Jin HR, Lee HS, Yeon JY, et al. Residual diplopia after repair of pure orbital blowout fracture: the importance of extraocular muscle injury. Am J Rhinol 2007; 21: 276-280.

24) Lyon DB and Newman SA. Evidence of direct damage to extraocular muscles as a cause of diplopia following orbital trauma. Ophthal Plast Reconstr Surg 1989; 5: 81-91. 
Fig. 1

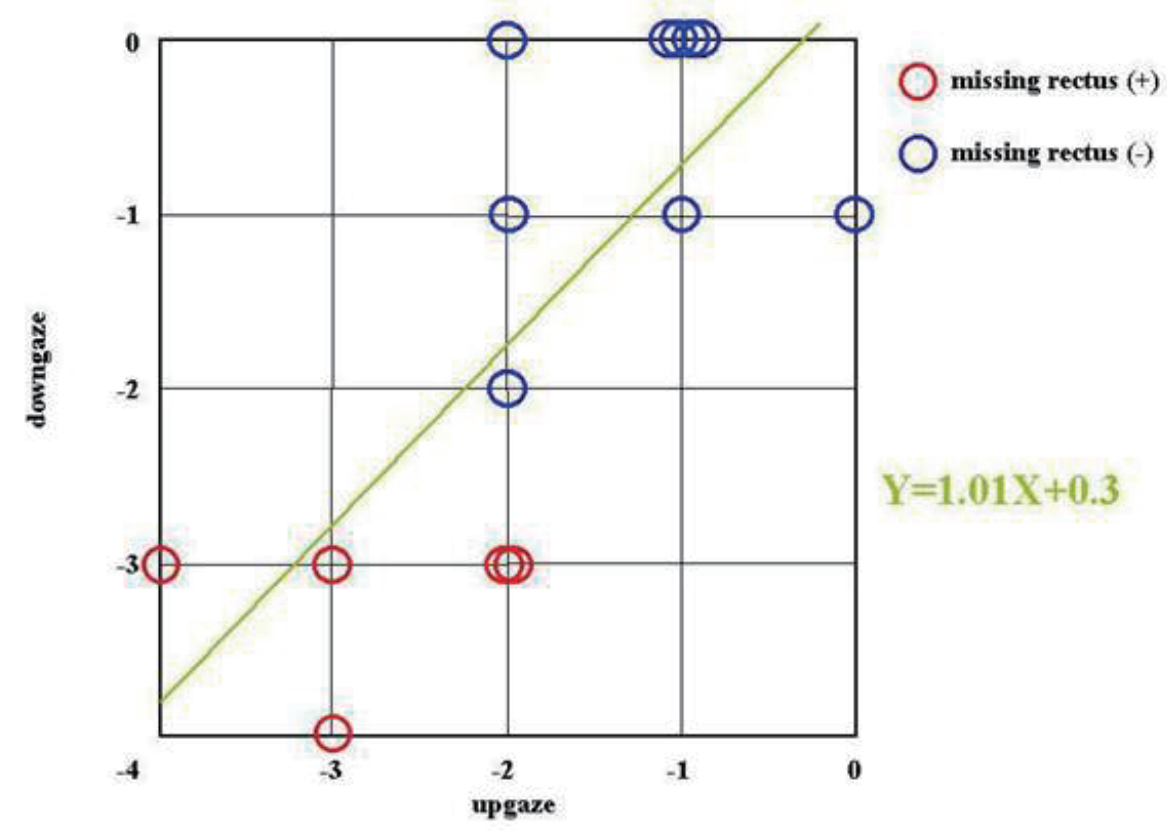

Fig. 2

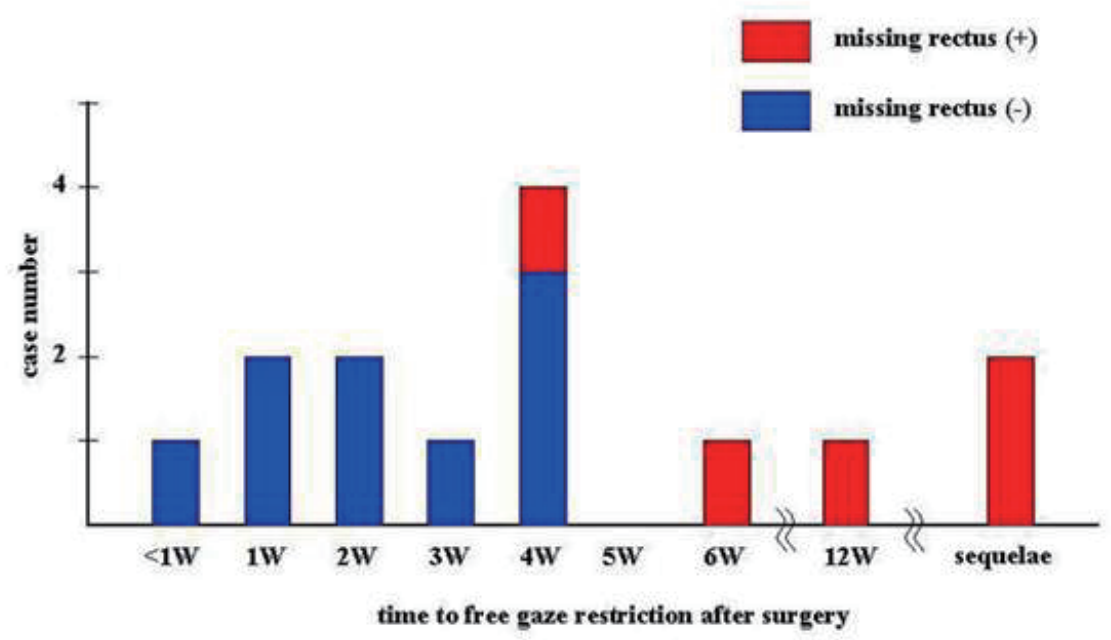


Fig. 3
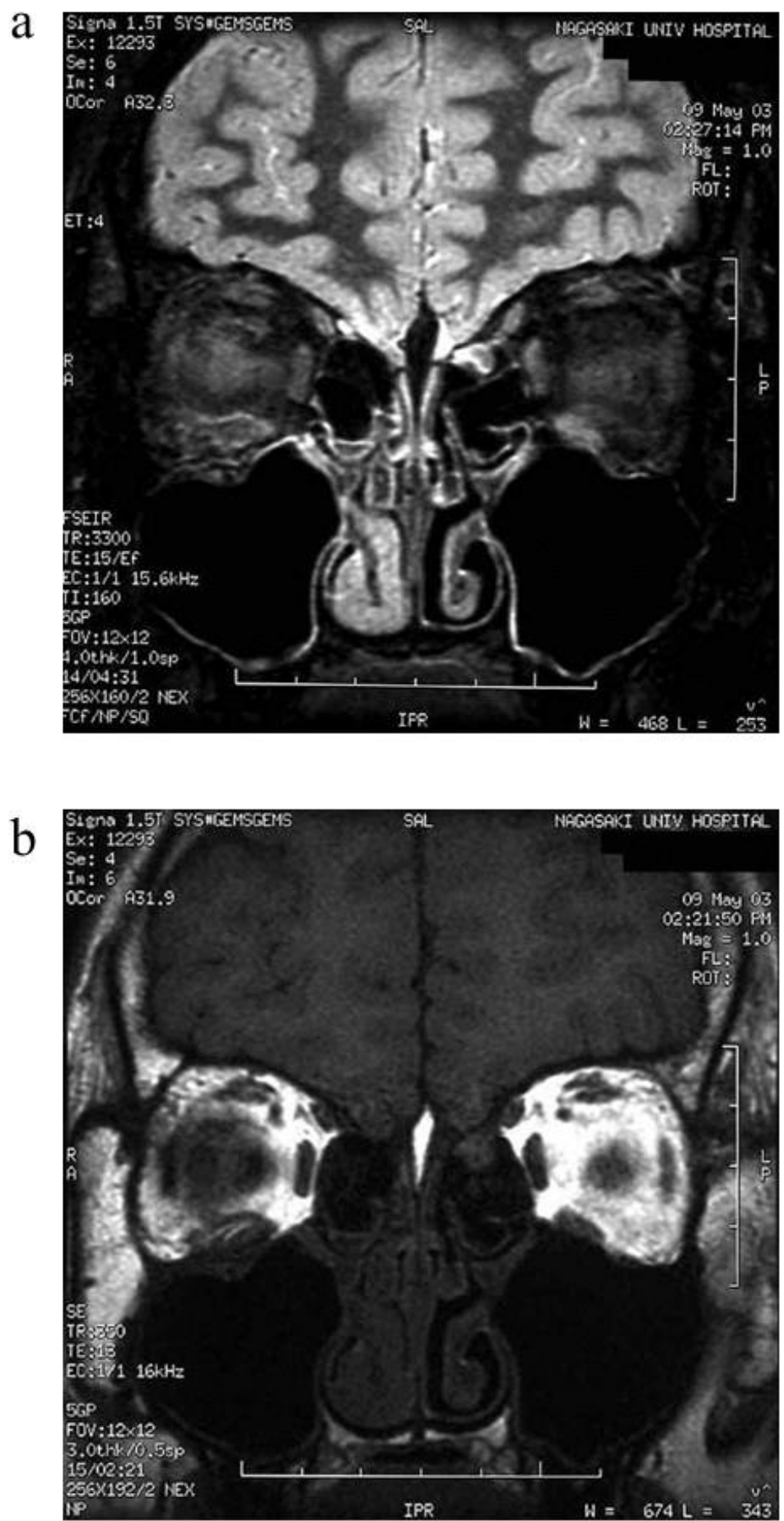
Fig. 4
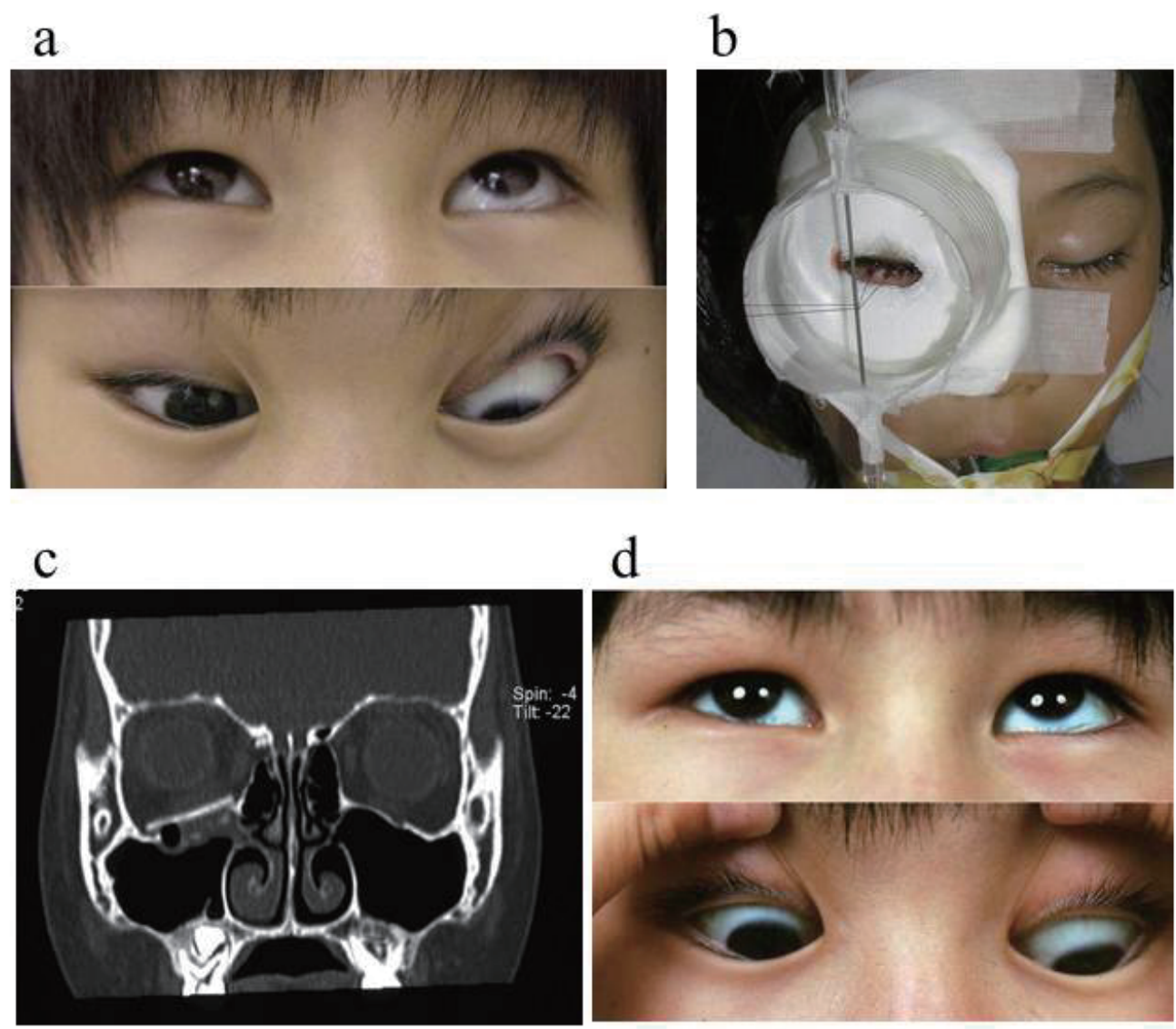

d

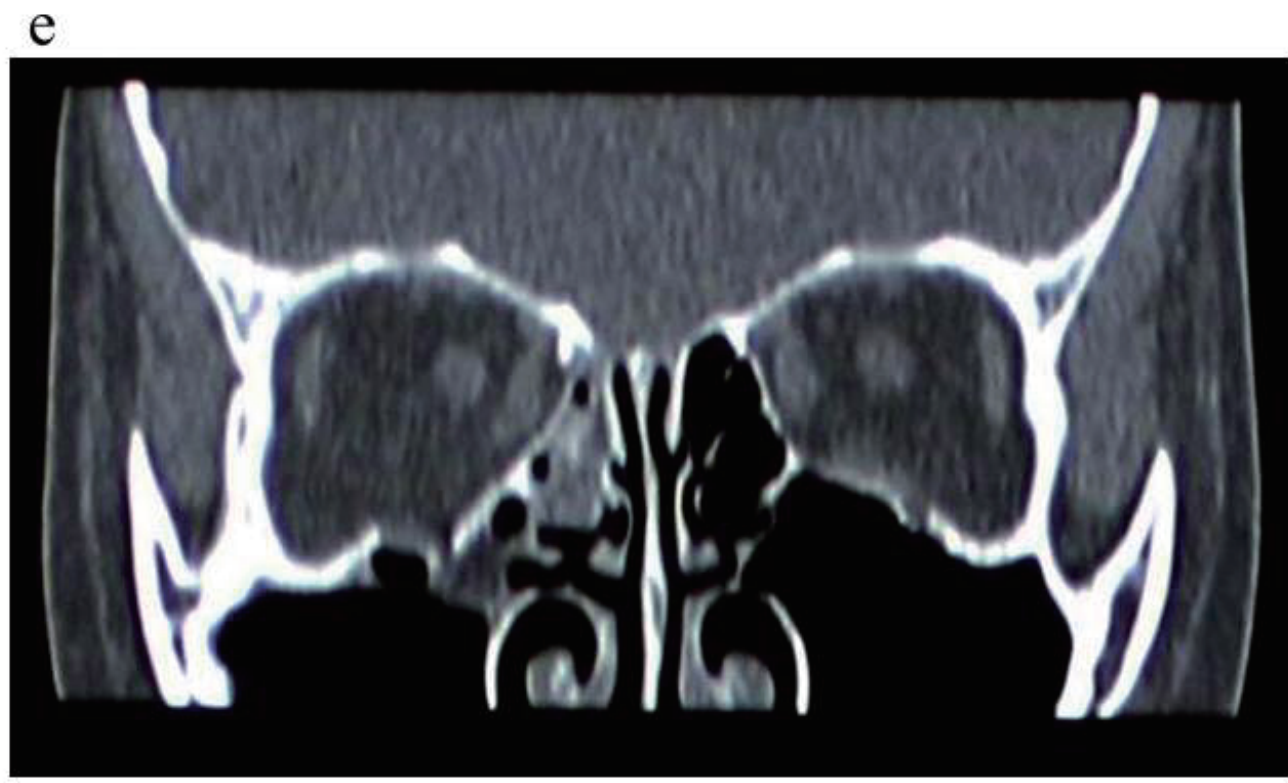


Fig. 5

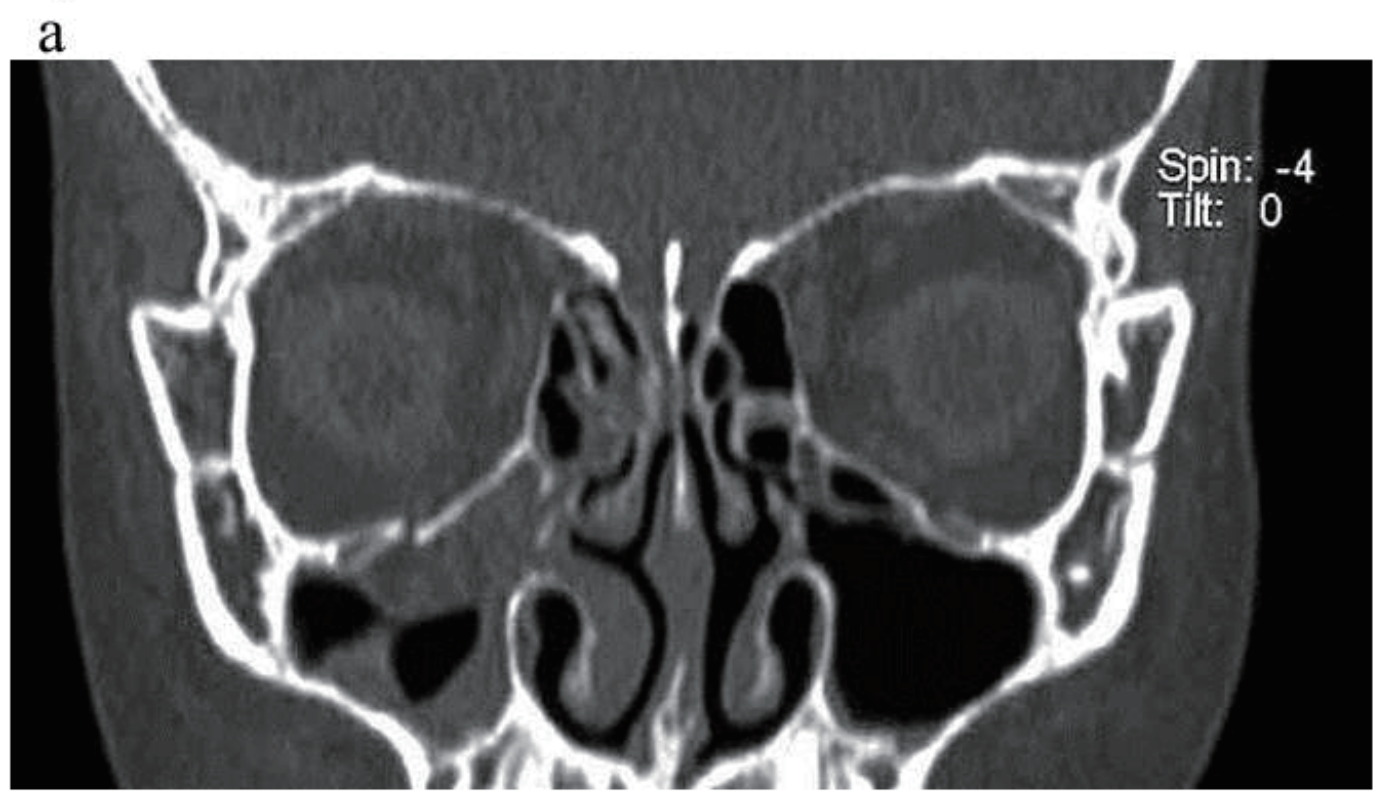

b

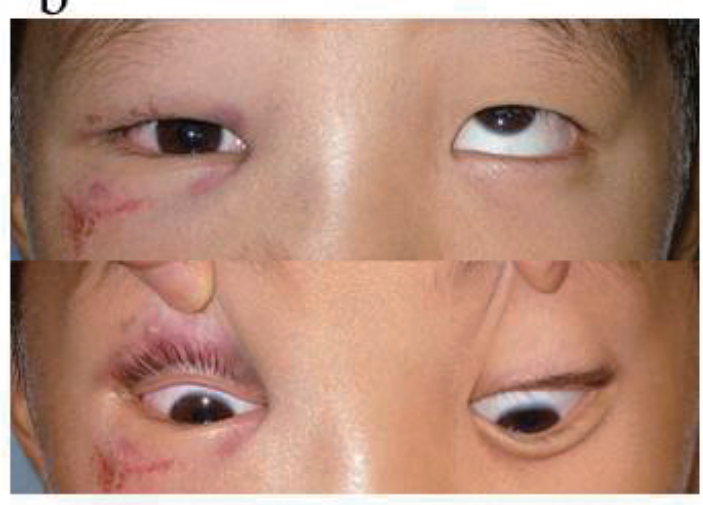

d

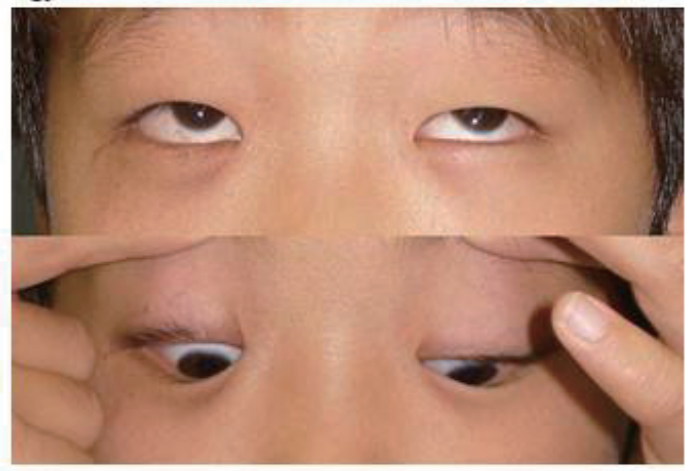

c

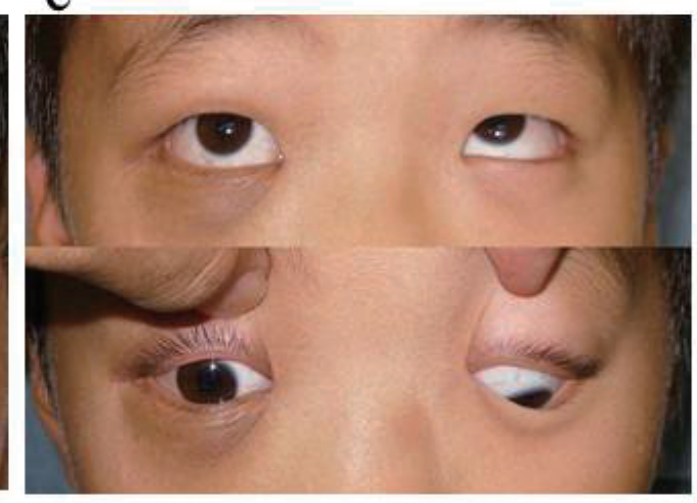

e

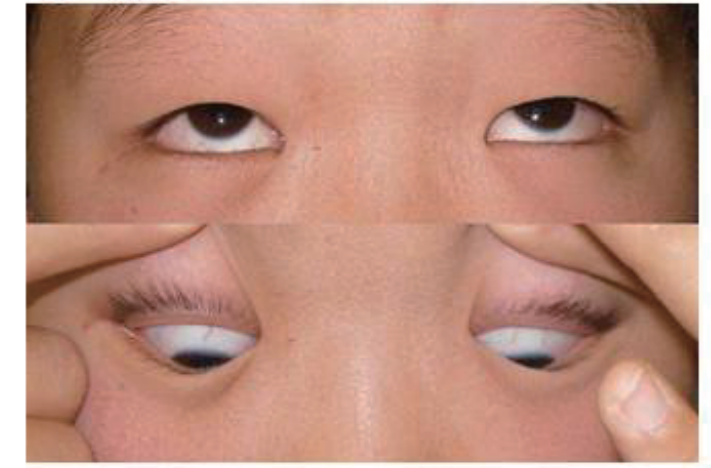


Fig. 6

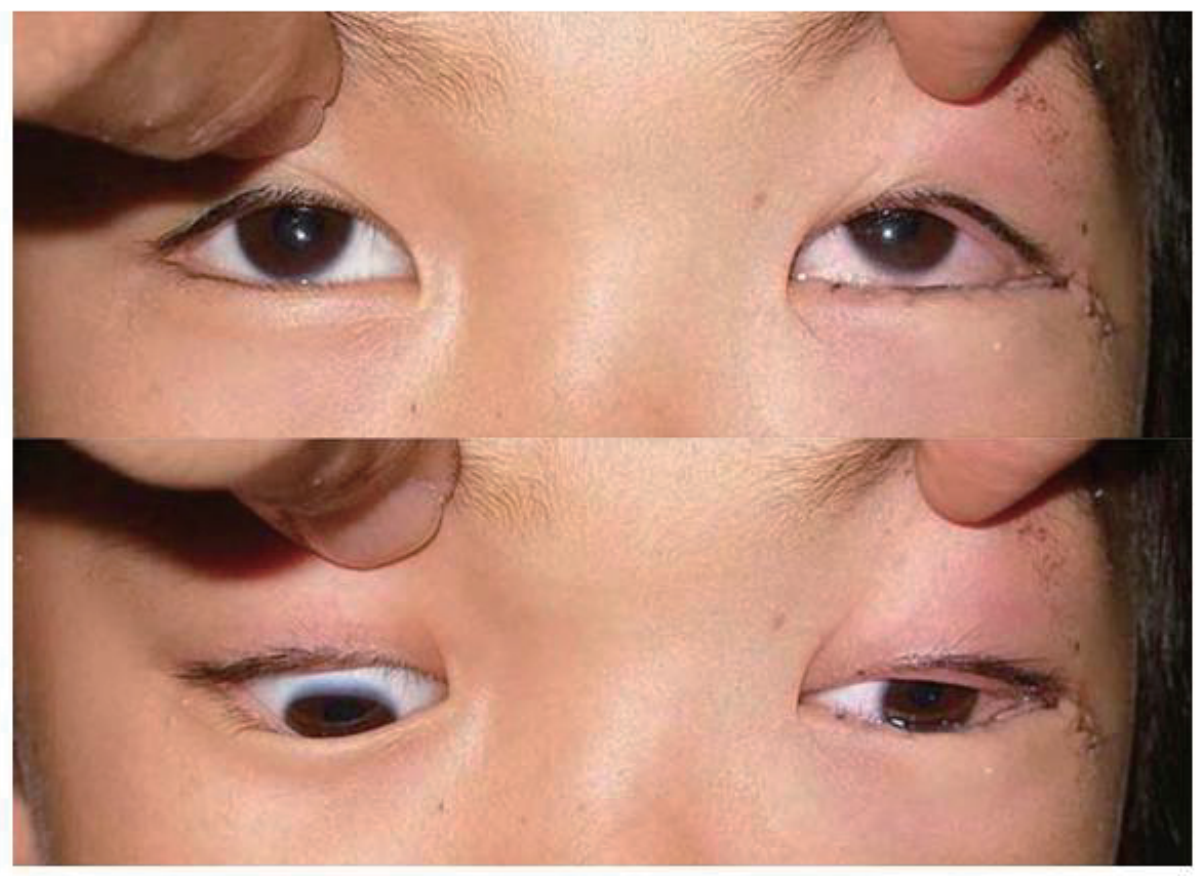


Fig. 7

a

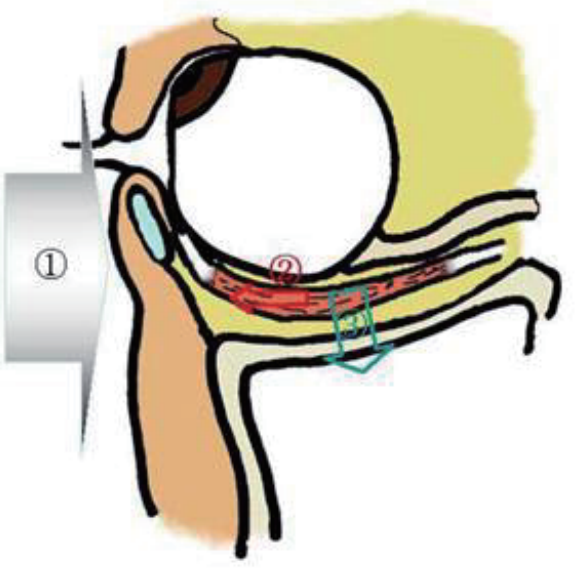

b

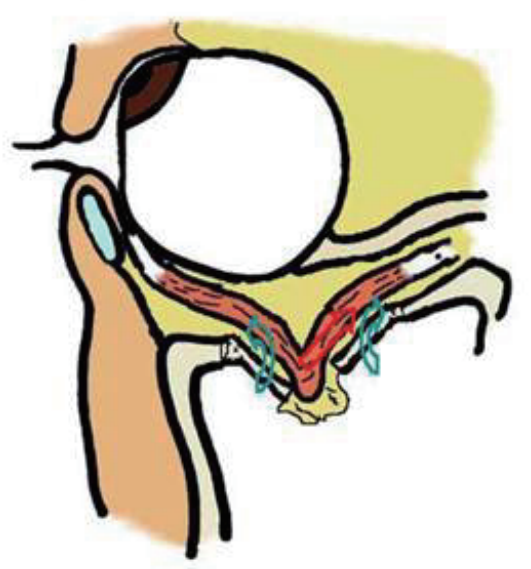

c

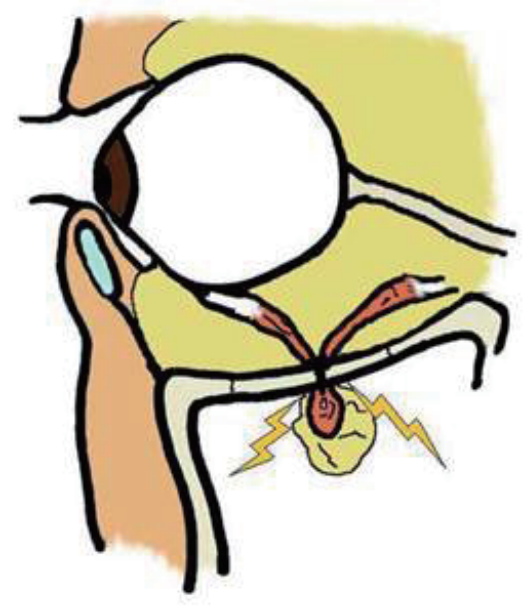


Table 1 . Clinical summary of 14 operative cases.

\begin{tabular}{|c|c|c|c|c|c|c|c|c|c|c|c|c|c|c|c|}
\hline & \multirow{2}{*}{$\begin{array}{c}\operatorname{sgo} \\
\text { (years) }\end{array}$} & \multirow{2}{*}{ sex } & \multirow{2}{*}{ canse } & \multicolumn{2}{|c|}{ CT fiadieg: } & \multirow{2}{*}{$\begin{array}{l}\text { time (days) to } \\
\text { preseatation }\end{array}$} & \multicolumn{2}{|c|}{ complaint } & \multicolumn{2}{|c|}{ motility } & \multirow{2}{*}{\begin{tabular}{|r|} 
time (days) to \\
sargery
\end{tabular}} & \multirow{2}{*}{ sargery } & \multirow{2}{*}{$\frac{\text { time to }}{\text { recorery }}$} & \multicolumn{2}{|c|}{ stmusax } \\
\hline & & & & fractare & MIR & & eapphtbalnoos & diplopia & घp & dowra & & & & preop & poutop \\
\hline case 1 & 10.9 & $\mathrm{~m}$ & sports & Sinse: & + & 1 & - & + & -3 & -4 & 8 & $O R+B G$ & 1squalost4 & - & - \\
\hline case 2 & 8.4 & $\mathrm{~m}$ & maves? & Enow & - & 3 & - & + & -1 & 0 & 12 & $O R+B G$ & 4 noots & - & - \\
\hline case 3 & 24.3 & $\mathrm{~m}$ & ipoets & Soser: & - & 0 & - & + & 0 & -1 & 10 & $O R+B G$ & 1 nest & $\mathrm{V} 2$ & $\mathrm{v} 2$ \\
\hline case 4 & 9.1 & $f$ & falling & Simos: & - & 2 & - & + & -2 & 0 & 10 & $O R+B G$ & 2 neots & - & - \\
\hline case 5 & 11.2 & $\mathrm{~m}$ & falling & Sinour & - & 10 & $\cdot$ & + & -1 & 0 & 16 & $O R+B G$ & 1 nesk & - & $\cdot$ \\
\hline case 6 & 17.0 & $\mathrm{~m}$ & wports & Sinos: & - & 7 & - & + & -2 & -1 & 12 & $O R+B G$ & 4 mosts & $\mathrm{V} 2$ & - \\
\hline case 7 & 16.4 & $\mathrm{~m}$ & anast: & Sinour & + & 2 & $\cdot$ & + & -2 & -3 & 5 & $O R+B G$ & 4 nestes & $\mathrm{V} 2$ & $\mathrm{~V}_{2}$ \\
\hline case 8 & 18.8 & $\mathrm{~m}$ & sports & Sinse: & - & 1 & $\cdot$ & + & -1 & -1 & 8 & $O R+B G$ & 2 mostes & $\cdot$ & $\mathrm{v} 2$ \\
\hline case 9 & 9.8 & $\mathrm{~m}$ & sports & Soser & $\cdot$ & 1 & - & + & -1 & 0 & 5 & $\mathrm{OR}$ & 3 dxys & - & $\cdot$ \\
\hline case $10^{*}$ & 69 & $f$ & sports & Soser & + & 20 & $\cdot$ & + & -2 & -3 & 24 & $O R+B G$ & 1squaloste & $\cdot$ & $\cdot$ \\
\hline case 11 & 10.9 & $\mathrm{~m}$ & yports & Sinos: & $\cdot$ & 4 & - & + & -2 & -2 & 11 & $O R+B G$ & 4 mosts & $\cdot$ & $\cdot$ \\
\hline case 12 & 8.7 & $\mathrm{~m}$ & falling & Soser: & + & 0 & $\cdot$ & + & -3 & -3 & 0 & $O R+B G$ & 6 moots & - & - \\
\hline case 13 & 7.6 & $m$ & falling & Sinas & $\cdot$ & 7 & - & + & -1 & 0 & 13 & $O R+B G$ & 4 mosis & $\cdot$ & $\cdot$ \\
\hline case 14 & 9.8 & $\mathrm{~m}$ & falling & Sinour & + & 2 & - & + & -4 & -3 & 2 & $O R+B G$ & 12 mesiss & - & - \\
\hline \multicolumn{16}{|c|}{ 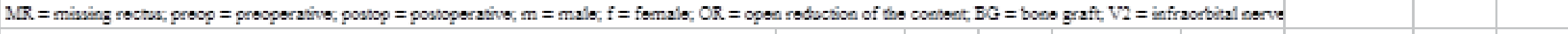 } \\
\hline \multicolumn{16}{|c|}{ 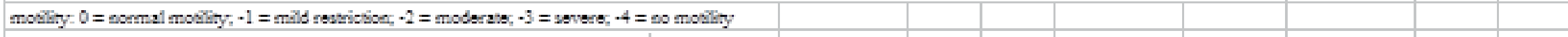 } \\
\hline \multicolumn{16}{|c|}{ "The data for case 10 were bevod on the first operavion at the previous bouptal | } \\
\hline \multicolumn{16}{|c|}{ 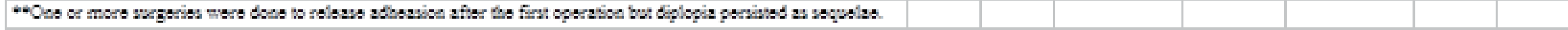 } \\
\hline
\end{tabular}


Table 2. Time to surgery from injury with or without "missing rectus"

\begin{tabular}{|l|r|r|r|r|r|}
\hline \multicolumn{7}{|c|}{ time to surgery (days) } \\
\hline & average* & $\min$ & $\max$ & \multicolumn{1}{c|}{$\mathrm{SD}$} & median \\
\hline missing rectus (+) & 7.8 & 0 & 24 & 9.45 & 5 \\
\hline missing rectus (-) & 10.8 & 5 & 16 & 3.11 & 11 \\
\hline & $* \mathrm{P}=0.397$ by Student's $t$-test. & & \\
\hline
\end{tabular}




\section{Figure Legends}

Fig. 1 Scatter diagram of preoperative ocular motility upon upgaze and downgaze in 14 surgical cases. The simple regression line is indicated in green $(\mathrm{r}=0.730, \mathrm{p}<0.003)$. Red circles denote cases with "missing rectus" and blue circles denote cases without "missing rectus" in CT findings. (With missing rectus vs without missing rectus; upgaze $p$-value $=0.0093$ and downgaze $\mathrm{p}$-value $=0.0027$ by the Mann-Whitney $U$ test.)

Fig. 2 Time of surgery to recovery. Red bar indicates cases with "missing rectus" and blue indicates cases without "missing rectus" in CT findings. In cases without "missing rectus" diplopia dissolved within one month but in cases with "missing rectus," diplopia was protracted more than four weeks.

Fig. 3 MRI images six months after surgery (a; STIR, b; T1). Surgery was performed eight days after injury, but restriction of the eye movement continued. Herniation was reduced but fibrous degeneration was seen in and around the inferior rectus muscle. After that, dissection was performed to release the fibrosis, but diplopia persisted. 
Fig. 4 A case with missing rectus and persistent diplopia. Surgical intervention was performed 20 days after injury, but the vertical restriction of eye movement persisted. The patient was referred to us for further treatment after three months. (a) Eye movement before our operation. Motility was -1 in upgaze and -2 in downgaze. (b) Just after our operation. After perimuscular dissection, we applied traction to the inferior rectus muscle for one week. (c) Coronal CT after operation. The floor was reconstructed with a large bone. (d) Eight months after our surgery. Motility improved (upgaze, -1; downgaze, -1 ), but diplopia continued. (e) The CT images just after injury. The right inferior rectus muscle disappeared above the floor.

Fig. 5 A case with missing rectus but without sequelae. (a) Coronal CT image before surgery. The right inferior rectus muscle partially disappeared above the floor. (b) Preoperative gaze restriction. (upgaze, -4; downgaze, -3) Two days after injury the patient presented with a blowout fracture and underwent an emergency operation. The patient did not experience nausea 
or vomiting from the time of injury until our inspection. (c) Two weeks after the operation. Supraduction was well recovered but infraduction was poor. A monoculus was worn on the left eye for one hour, three times a day, to exercise the right affected eye. (upgaze, -1; downgaze, -3) (d) Eight weeks after the operation. (upgaze, 0; downgaze, -1) (e) It took twelve weeks until the vertical restriction subsided.

Fig. 6 Paradoxical eye movement after the blowout surgery (Case 12, three days after surgery). Supraduction of the affected eye was superior to that of the intact eye in the early phase after the operation. This overactivity of the affected side was also occasionally observed after late surgery.

Fig. 7 Role of Bell's phenomenon in the linear-type blowout fracture. (a) Almost all patients close their eyes firmly at injury, and the supraduction caused by Bell's phenomenon results in lengthening of the inferior rectus. The muscle is brought to the front of the eye, and to frequent sites of fractures. (b, c) After breaking the floor, the muscle is herniated through the fracture site. Then the door is closed by the force of its elasticity. More 
damage may occur upon closing the "trap door" by the returning force which may assist closing the door when the eye returns to the normal position. (For a better understanding, the fracture line is depicted in the coronary direction. Clinically most linear-type fractures in the floor occur in the sagittal direction.) 